\title{
Identificación de normas y directrices para bibliotecas: las unidades de información
}

\author{
Yadira Rosario Nieves Lahaba*
}

Artículo recibido:

27 de octubre de 2017

Artículo aceptado:

15 de octubre de 2018

Artículo de investigación

\section{Resumen}

Las unidades de información han utilizado la calidad como un concepto inherente a sus actividades. El uso de normas se avala en aras de garantizar una actuación cercana a las necesidades de su público objetivo. Los objetivos de este trabajo se enmarcan en identificar documentos que regulan y orientan los procesos en este tipo de organizaciones, así como explorar la opinión de profesionales involucrados con la actividad bibliotecológica con respecto al uso de estándares en las unidades de información. Los resultados de la encuesta realizada a estos profesionistas arrojan que se considera la aplicación de normas como un proceso necesario.

* $\quad$ Facultad de Filosofía y Letras, Universidad Autónoma de Nuevo León, México yadira.nieveslahaba@gmail.com INVESTIGACIÓN BIBLIOTECOLÓGICA, vol.33, núm.78, enero/marzo, 2019, México, ISSN: 2448-8321 pp. 81-103 
Palabras clave: Calidad para Bibliotecas; Normas Mexicanas; Normas Internacionales; Gestión de la Calidad

Identification of norms and guidelines for libraries; the units of information

Yadira Rosario Nieves Lababa

\section{Abstract}

The information units have used quality as an inherent concept to their activities. The use of standards has the purpose of guaranteeing an action close to the needs of its target audience. The finality of this word is to identify normative documents which regulate and guide the processes of this kind of information units, and also to explore the opinion of professionals involved in the library science activity, with respect to the use of standards in the information units. The answers of the survey made upon these professionals hold that the application of norms is considered as a necessary process.

Keywords: Quality of Libraries; Mexican Standards; International Standards; Quality Management

\section{INTRODUCCIÓN}

T as unidades de información están inmersas en una realidad que demanda Lprocesos más complejos (Aenor, 2005; Amante, Costa y Extremeño, 2012; Arias, 2013; Arriola Navarrete, 2017; Cárdenas Zardoni, 2012; López Lemos, 2016; Moreno Jiménez, 2005; Pinto Molina y Fernández Valdés, 2010), por lo que la calidad con que se lleven a cabo sigue siendo un factor esencial.

Un argumento aceptado, en este contexto, remarca que la calidad es determinada y enunciada por el usuario (Arias, 2013; Balagué y Saarti, 2014; Pinto Molina y Fernández Valdés, 2010). Los procesos técnicos y organizativos que se llevan a cabo para realizar procesos, prestar un servicio o desarrollar un producto son actividades que no sólo deben estar regidas por normas, sino también por las necesidades y satisfacción de aquéllos (Arias, 2013; Balagué y Saarti, 2014; Pinto Molina y Fernández Valdés, 2010). 
En este ámbito, emergen otros factores que están influyendo en los procesos actuales de este tipo de instituciones y en la calidad con la que se desempeñan, como la evolución de las tecnologías (Ávila Barrientos, 2016; Pinto Molina y Fernández Valdés, 2010; Linares Herrera, 2010), el creciente desarrollo en las habilidades de los usuarios (Arriola Navarrete, 2017; Cárdenas Zardoni, 2012; Pinto Molina y Fernández Valdés, 2010), el cambio en las necesidades de información (Ávila Barrientos, 2016; Cárdenas Zardoni, 2012; Pinto Molina y Fernández Valdés, 2010), la proliferación de la educación en línea (Arriola Navarrete, 2017; Rosas de Maidana, 2013; Pinto Molina y Fernández Valdés, 2010), el acceso abierto a la información (Rosas de Maidana, 2013; Ávila Barrientos, 2016), el paradigma de la sostenibilidad (Vargas Echeverría, 2017) y las leyes de transparencia en la información (Pacios, 2016).

Ante esta situación, resulta interesante preguntarse qué normas y directrices rigen la actividad de las unidades de información y cuál es la opinión de especialistas que trabajan en estas instituciones sobre el uso de normas y directrices. En este trabajo, las directrices hacen referencia a todos aquellos parámetros que regulan la actividad de las entidades, en cambio las normas hacen referencia a aspectos técnicos que indican cómo debe ser un proceso o cómo debe funcionar un servicio. Ambos aspectos, normas y directrices, son considerados orientadores de la calidad.

\section{Normativas de uso internacional}

Varios son los organismos que orientan y norman los procesos de las unidades de información. Muchos de estos organismos son especializados en un área específica del conocimiento mientras que otros abordan diferentes áreas. La Asociación Española de Normalización y Certificación (Aenor) ha emitido varias normas relacionadas con la actividad en unidades de información, algunas de las cuales se describen en la Tabla 1.

\begin{tabular}{|l|l|}
\hline \multicolumn{1}{|c|}{ Código } & \multicolumn{1}{c|}{ Título de la norma } \\
\hline UNE-CEN/TS 16371:2012 EX & $\begin{array}{l}\text { Directrices para la implementación de las normas 15744 y EN } \\
15907\end{array}$ \\
\hline UNE-EN 15744:2011 & $\begin{array}{l}\text { Identificación de películas. Conjunto mínimo de metadatos para } \\
\text { trabajos cinematográficos }\end{array}$ \\
\hline UNE-EN 15907:2011 & $\begin{array}{l}\text { Identificación de películas. Mejora de la interoperabilidad de los } \\
\text { metadatos. Conjuntos y estructuras de elementos }\end{array}$ \\
\hline UNE 93200:2008 & Cartas de servicios. Requisitos \\
\hline UNE 50137:2000/1M:2005 & $\begin{array}{l}\text { Información y documentación. Indicadores de rendimiento } \\
\text { bibliotecario }\end{array}$ \\
\hline
\end{tabular}


Tabla 1. Algunas normas Aenor relacionadas con la actividad en las unidades de información

El contenido de estas normas está relacionado en gran medida con los procesos bibliotecológicos para la organización y evaluación de la información. Por ejemplo: la norma 16371 orienta acerca de los enfoques tecnológicos que se deben utilizar en la aplicación de las normas 15744 y 15907 al momento de asignar metadatos (Aenor, 2012). Otro ejemplo es el referente a la norma 50137, la cual se corresponde con la norma ISO 11620 (Tabla 2), donde se abordan indicadores de evaluación en relación con cuatro ejes: recursos, acceso e infraestructura, uso, eficiencia potencial y desarrollo (Aenor, 2005).

Un caso interesante es la norma 93200, la cual no es específica para bibliotecas; sin embargo, aborda aspectos que resultan de gran utilidad para informar a los ciudadanos sobre los servicios que se ofrecen y sobre los derechos que les asisten en relación con los compromisos de calidad (Aenor, 2008).

Aenor ha trabajado en conjunto con la Organización Internacional para la Estandarización (ISO) cubriendo una amplia gama de áreas y procesos (Tabla 2), tales como organización y almacenamiento de documentos físicos y digitales, préstamos interbibliotecarios, evaluación y conservación de documentos, ofreciendo un marco de referencia para establecer el plan de desarrollo y la mejora continua de la calidad organizacional (Texidor, 2009). En este sentido, en la Tabla 2 se describen algunas normas ISO que resultan significativas, entre ellas se encuentran la ISO 9001, ISO 2789, ISO 11620, ISO 15489 y la ISO 30300.

\begin{tabular}{|l|l|}
\hline \multicolumn{1}{|c|}{ Código } & \multicolumn{1}{c|}{ Título de la norma } \\
\hline ISO 16439:2014 & $\begin{array}{l}\text { Información y documentación - Métodos y procedi- } \\
\text { mientos para evaluar el impacto de las bibliotecas }\end{array}$ \\
\hline IS0 15489 & $\begin{array}{l}\text { Información y documentación - Gestión de docu- } \\
\text { mentos Parte 1 y 2 }\end{array}$ \\
\hline IS0 30300 & $\begin{array}{l}\text { Información y documentación. Sistemas de gestión } \\
\text { para los documentos. Fundamentos y vocabulario }\end{array}$ \\
\hline ISO 9001:2015 & Sistemas de Gestión de la Calidad \\
\hline ISO 11799:2015 & $\begin{array}{l}\text { Información y documentación - Requisitos de } \\
\text { conservación de documentos para los materiales de } \\
\text { archivos y bibliotecas }\end{array}$ \\
\hline ISO 10160:2015 & $\begin{array}{l}\text { Información y documentación - Interconexión de sis- } \\
\text { temas abiertos - Definición del servicio de solicitud } \\
\text { de préstamo interbibliotecario }\end{array}$ \\
\hline
\end{tabular}




\begin{tabular}{|l|l|}
\hline ISO 18626:2014 & $\begin{array}{l}\text { Información y documentación - Las operaciones de } \\
\text { préstamo entre bibliotecas }\end{array}$ \\
\hline IS0 11620:2014 & $\begin{array}{l}\text { Información y documentación - Indicadores de } \\
\text { rendimiento de las bibliotecas }\end{array}$ \\
\hline IS0 10161-1:2014 & $\begin{array}{l}\text { Información y documentación - Interconexión de } \\
\text { sistemas abiertos - Solicitud de préstamo interbi- } \\
\text { bliotecario Especificación del protocolo - Parte 1: } \\
\text { Especificación del protocolo, Parte 2: Declaración } \\
\text { de conformidad de implementación de protocolo } \\
\text { (PICS) }\end{array}$ \\
\hline IS0 2789:2014 & $\begin{array}{l}\text { Información y documentación - Estadísticas interna- } \\
\text { cionales de bibliotecas }\end{array}$ \\
\hline IS0/TR 14873:2013 & $\begin{array}{l}\text { Información y documentación - Estadísticas y } \\
\text { cuestiones de calidad para el archivo web }\end{array}$ \\
\hline ISO/TR 11219:2012 & $\begin{array}{l}\text { Información y documentación - Condiciones cualita- } \\
\text { tivas y estadísticas básicas para la construcción de } \\
\text { bibliotecas - Espacio, la función y el diseño }\end{array}$ \\
\hline IS0/TR 28118: 2009 & $\begin{array}{l}\text { Información y documentación - Indicadores de } \\
\text { rendimiento para bibliotecas nacionales }\end{array}$ \\
\hline ISO 23081-1 & $\begin{array}{l}\text { Información y documentación - Proceso de gestión } \\
\text { de documentos. Metadatos para la gestión de } \\
\text { documentos }\end{array}$ \\
\hline
\end{tabular}

Tabla 2. Resumen de normas ISO relacionadas con la actividad de las ciencias de la información

La ISO 2789 orienta sobre estadísticas internacionales en relación a "recursos y productos de la biblioteca en términos de tamaño de la colección, número y tipos de usuarios, uso de los servicios, finanzas, personal y espacio" (Albelda et al., 2014: 13-14), estos indicadores son básicos para la comparación cronológica dentro de la misma biblioteca y entre bibliotecas (Aenor, 2004). De igual manera, la ISO 11620 incorpora indicadores de rendimiento para bibliotecas nacionales del Informe Técnico ISO/TR 28118:2009, abordando aspectos como la "digitalización, repositorios institucionales para recursos electrónicos, preservación, y cooperación en asociaciones y proyectos" (Albelda et al., 2014: 8). El uso de los indicadores que propone esta norma constituye una referencia esencial para la medición de procesos, recursos y servicios en relación con eficiencia, adecuación, uso y disponibilidad (Gaminde, Martínez Hervás y Yúfera Rodríguez, 2015; González Guitián y Molina Piñeiro, 2008).

En cambio, la ISO 15489 aborda las responsabilidades en un sistema de gestión documental y resalta la importancia de aspectos como la organización y el cumplimiento de las normas, la consideración de los documentos como plena prueba o el rendimiento de la organización (Giménez Chornet, 2015; Santovenia Díaz y Cañedo Andalia, 2006). Todo esto para garantizar 
la creación, captura y administración, autenticidad, fiabilidad, integridad y disponibilidad en la gestión documental, teniendo en cuenta el contexto de la organización (ISO, 2016).

Otros documentos que contribuyen a normalizar la gestión documental son metadatos (UNE-ISO 23081), análisis de procesos de trabajo (ISO/TR 26122), formatos de fichero de documento electrónico (UNE-ISO 19005), guías de implementación para digitalización de documentos (UNE-ISO/TR 13028) o requerimientos funcionales para documentos electrónicos (ISO 16175) (Alsina, 2012). En este sentido, la nueva versión de la norma 9001:2015 también aporta elementos que facilitan la optimización del sistema documental (López Lemos, 2016).

Por otra parte, la norma ISO 30300 facilita toda la terminología necesaria para la aplicación de los requisitos del sistema de gestión de documentos (Alsina, 2012). La norma se fundamenta en el enfoque de procesos, el rol de la alta dirección y las relaciones con los otros sistemas de gestión (Aenor, 2011)

Aenor e ISO han trabajado para desarrollar una amplia gama de documentos normativos. Estos documentos no sólo aportan estrategias para la creación e implantación de sistemas, también realizan contribuciones sólidas a la actividad bibliotecológica. Algunos de estos documentos se describen en la Tabla 3.

\begin{tabular}{|l|l|}
\hline \multicolumn{1}{|c|}{ Código } & \multicolumn{1}{c|}{ Título de la norma } \\
\hline UNE-ISO 2789:2014 & $\begin{array}{l}\text { Información y documentación. Estadísticas interna- } \\
\text { cionales de bibliotecas }\end{array}$ \\
\hline UNE-ISO 690:2013 & $\begin{array}{l}\text { Información y documentación. Directrices para la } \\
\text { redacción de referencias bibliográficas y de citas de } \\
\text { recursos de información }\end{array}$ \\
\hline UNE-ISO 14416:2013 & $\begin{array}{l}\text { Información y documentación. Requisitos para la } \\
\text { encuadernación de libros, publicaciones periódicas, } \\
\text { publicaciones seriadas y otros documentos en papel } \\
\text { para la utilización en archivos y bibliotecas. Métodos } \\
\text { y ensayos }\end{array}$ \\
\hline UNE-ISO 15836:2011 & $\begin{array}{l}\text { Información y documentación. Conjunto de elemen- } \\
\text { tos de metadatos Dublín Core. (ISO 15836:2009) }\end{array}$ \\
\hline UNE-ISO 22310:2010 & $\begin{array}{l}\text { Información y documentación. Directrices para los } \\
\text { redactores de normas para el establecimiento de } \\
\text { los requisitos de la gestión de documentos en las } \\
\text { normas }\end{array}$ \\
\hline UNE-ISO 5127:2010 & \begin{tabular}{l} 
Información y documentación. Vocabulario \\
\hline UNE-ISO 17933:2006
\end{tabular} \\
\hline UNE-ISO 2108:2006 & $\begin{array}{l}\text { GEDI. Intercambio de documentos electrónicos } \\
\text { genérico. (ISO 17933,2000) }\end{array}$ \\
\hline & $\begin{array}{l}\text { Información y documentación. Número normalizado } \\
\text { internacional del libro (ISBN). (ISO 2108,2005) }\end{array}$ \\
\hline
\end{tabular}




\begin{tabular}{|l|l|}
\hline UNE-ISO 22310:2010 & $\begin{array}{l}\text { Información y documentación. Directrices para los } \\
\text { redactores de normas para el establecimiento de } \\
\text { los requisitos de la gestión de documentos en las } \\
\text { normas }\end{array}$ \\
\hline UNE-ISO 2709:2006 & $\begin{array}{l}\text { Documentación e información. Formato para el } \\
\text { intercambio de información }\end{array}$ \\
\hline UNE-ISO 3297:2008 & $\begin{array}{l}\text { Información y documentación. Número internacional } \\
\text { normalizado de publicaciones en serie (ISSN) }\end{array}$ \\
\hline
\end{tabular}

Tabla 3. Resumen de normas desarrolladas por Aenor e Iso relacionadas con la actividad en las unidades de información

Sin embargo, también hay que señalar que hay áreas de oportunidad pues es común encontrar reincidencia excesiva de conceptos ya explicitados y tratados en otras normas, como es el caso de la recientemente publicada norma ISO 15489:2016 (Tabla 2) donde se establece una estrecha relación con la norma ISO 30300. Otros de los organismos que han realizado alianzas son la Organización de las Naciones Unidas para la Educación, la Ciencia y la Cultura (Unesco) y la Federación Internacional de Asociaciones de Bibliotecarios y Bibliotecas (IFLA). Si bien es cierto que estas organizaciones no establecen normas, es posible afirmar que han emitido directrices con el objetivo de orientar la creación y el funcionamiento de las bibliotecas públicas, escolares y digitales en todo el mundo.

La Unesco, en el Manifiesto sobre la Biblioteca Pública aprobado en 1994, el cual aparece en su página web, proclama la confianza en este tipo de institución al definir a las bibliotecas como el motor de la educación, cultura e información y como representante esencial para el fomento de la paz y la espiritualidad del ser humano (Unesco, s. a.).

Por otra parte, en el Manifiesto sobre la Biblioteca Escolar, resultado de la colaboración entre IFLA y Unesco (IFLA, 1999), se define y potencia el papel de las bibliotecas escolares con el objetivo de permitir a los estudiantes adquirir las herramientas y contenidos de aprendizaje que les posibiliten desarrollar plenamente sus capacidades, de forma que sean capaces de seguir aprendiendo durante toda su vida y tomar decisiones informadas.

En el Manifiesto sobre las Bibliotecas Digitales, IFLA y la Unesco declaran que "La brecha digital es una brecha de información. Subsanar la brecha digital es un factor clave para alcanzar los Objetivos de Desarrollo del Milenio de las Naciones Unidas. El acceso a los recursos informativos y los medios de comunicación contribuye a la salud y la educación, así como al desarrollo cultural y económico" (IFLA, s. a.). 
El contenido de estos tres documentos orienta respecto de los servicios a prestar, la función del bibliotecario, la responsabilidad social, las necesidades de información, las habilidades de los usuarios, el papel de la tecnología, la relación entre las bibliotecas y la necesidad de coordinarse para la colaboración en favor de la cultura, la información, la alfabetización y la educación, aspectos de gran vigencia y valor.

Otro aspecto en el que coinciden estos documentos es el que se refiere al acceso a la información, el cual merece especial atención por el coherente abordaje que los vincula. Para estas organizaciones resulta imprescindible que no se restrinja el acercamiento al conocimiento pues es fundamental para garantizar la construcción de un mundo equitativo actual y futuro.

Un aspecto muy significativo, pues tradicionalmente las bibliotecas han sido reconocidas como salvaguarda y no como productoras, es que en estos documentos se aborda la relación del bibliotecario con los docentes y la necesidad de trabajar en favor de facilitar el aprendizaje, así como la imperiosa necesidad de la calidad en la creación de contenidos, viendo la cultura no sólo como un elemento diverso a proteger sino como vía para el progreso, la expresión y el diálogo entre los seres humanos.

Las directrices de la IFLA y la Unesco constituyen herramientas indispensables para que los líderes de todo el mundo se comprometan con la actividad de las unidades de información. El contenido de estos documentos permite una relación flexible pero a la vez detallada de cómo garantizar y potenciar la calidad en el funcionamiento de estas entidades, así como otorgar reconocimiento a las actividades que realizan.

En este sentido, la Asociación Americana de Bibliotecas (American Library Association, ALA), la más antigua y grande en el mundo, también establece parámetros coherentes con su política general. ALA considera que estos parámetros constituyen políticas que representan la génesis de las bibliotecas a través de los valores compartidos. Por eso, la Asociación de Bibliotecas Universitarias y de Investigación (Association Of College And Research Libraries, ACRL), una de las 11 divisiones de la ALA, al establecer directrices (Tabla 4) ayuda a las bibliotecas, instituciones académicas y agencias de acreditación a comprender los componentes de las bibliotecas, las cuales son regularmente revisadas y actualizadas por sus miembros.

\begin{tabular}{|l|l|}
\hline \multicolumn{1}{|c|}{ Título } & \multicolumn{1}{c|}{ Estatus } \\
\hline $\begin{array}{l}\text { Marco de la Alfabetización de Información para la } \\
\text { Educación Superior }\end{array}$ & $\begin{array}{l}\text { Aprobado por el Consejo ACRL 11 de enero de } \\
2016\end{array}$ \\
\hline $\begin{array}{l}\text { Normas para los Servicios de las Bibliotecas para } \\
\text { la Educación a Distancia }\end{array}$ & Revisadas junio de 2016 \\
\hline
\end{tabular}




\begin{tabular}{|c|c|}
\hline $\begin{array}{l}\text { Declaración de ACRL sobre la Política de Acceso } \\
\text { Abierto }\end{array}$ & $\begin{array}{l}\text { Aprobada por el Consejo de Administración de la } \\
\text { ACRL durante la Conferencia Anual de ALA, junio } \\
\text { de } 2016\end{array}$ \\
\hline Declaración sobre la Libertad Académica & $\begin{array}{l}\text { Aprobada por el Consejo de Administración de la } \\
\text { ACRL durante la Conferencia Anual de ALA, junio } \\
\text { el año } 2015\end{array}$ \\
\hline $\begin{array}{l}\text { Directrices para los servicios bibliotecarios para } \\
\text { estudiantes universitarios no graduados }\end{array}$ & Aprobadas en octubre de 2013 \\
\hline $\begin{array}{l}\text { Guía: Características de los programas de alfabe- } \\
\text { tización informacional }\end{array}$ & Revisada en junio del 2013 \\
\hline $\begin{array}{l}\text { Objetivos de la Instrucción de Alfabetización } \\
\text { Informacional: un modelo de declaración para los } \\
\text { bibliotecarios académicos }\end{array}$ & $\begin{array}{l}\text { Aprobados por el Consejo ACRL de enero de } \\
2001\end{array}$ \\
\hline $\begin{array}{l}\text { Declaración sobre la certificación y licencias de } \\
\text { los bibliotecarios académicos }\end{array}$ & $\begin{array}{l}\text { Reafirmada por la Junta ACRL en el Comité Ejecu- } \\
\text { tivo de la reunión de primavera, } 11 \text { de mayo, } 2011\end{array}$ \\
\hline $\begin{array}{l}\text { Directrices para los bibliotecarios académicos Sin } \\
\text { Facultad de estado }\end{array}$ & $\begin{array}{l}\text { Aprobadas por el Consejo de Administración de la } \\
\text { ACRL el } 21 \text { de octubre } 2011\end{array}$ \\
\hline Normas para Bibliotecas en Educación Superior & $\begin{array}{l}\text { Aprobadas por el Consejo de Administración de la } \\
\text { ACRL, octubre de } 2011\end{array}$ \\
\hline $\begin{array}{l}\text { Guía para el nombramiento, ascenso de los } \\
\text { bibliotecarios }\end{array}$ & $\begin{array}{l}\text { Aprobada en la Conferencia Anual de ALA, junio } \\
\text { de } 2010\end{array}$ \\
\hline $\begin{array}{l}\text { Directrices para los Centros de Materiales } \\
\text { Curriculares }\end{array}$ & Aprobadas por la ACRL y ALA, enero de 2009 \\
\hline $\begin{array}{l}\text { Declaración ACRL/SAA conjunta sobre el acceso } \\
\text { a material de investigación en archivos y bibliote- } \\
\text { cas especiales }\end{array}$ & $\begin{array}{l}\text { Aprobada por el Consejo ACRL durante la Confe- } \\
\text { rencia Anual de ALA, julio de } 2009\end{array}$ \\
\hline $\begin{array}{l}\text { Directrices: Las competencias para los profesio- } \\
\text { nales de Colecciones Especiales }\end{array}$ & $\begin{array}{l}\text { Aprobadas por el Consejo ACRL } 1 \text { de julio de } \\
2008\end{array}$ \\
\hline
\end{tabular}

Tabla 4. Resumen de normas y directrices ACRL

\section{Breve acercamiento a las directrices en México}

El Consejo Nacional para Asuntos Bibliotecarios de las Instituciones de Educación Superior, A. C. de México (CONPAB-IES, A. C.) ha desarrollado las Normas para Bibliotecas de Instituciones de Educación Superior e Investigación (NBIESI). En estos documentos se establecen principios para la evaluación de los servicios bibliotecarios en México (CONPAB-IES y Verdugo Sánchez, 2012). En las NBIESI se establecen la gestión organizacional, la estructura constitutiva y la usabilidad informativa, y se desarrollan apartados referentes a la función de la biblioteca académica, organización, recursos humanos, recursos financieros, infraestructura, acervos, organización técnica de recursos documentales, servicios, formación de usuarios, evaluación. 
Además, contiene un apéndice para el tratamiento del acervo histórico universitario, asegurando de esta manera la protección y subsistencia de la memoria institucional para las futuras generaciones (CONPAB-IES y Verdugo Sánchez, 2012).

En relación con el apartado Servicios, el documento remarca los aspectos siguientes:

- La necesidad de difusión de los servicios de forma permanente, a través de materiales y publicaciones informativas.

- La importancia de satisfacer las necesidades de información de los usuarios, cumpliendo con las necesidades y características de cada institución en la que se desenvuelve.

- Cómo los servicios deben extenderse a la comunidad en general para no privarlos de conocimiento.

- La necesidad de que cada servicio cuente con un reglamento, el cual debe ser accesible para los usuarios.

Es indudable la importancia de las bibliotecas universitarias en la socialización de conocimientos y en la formación de competencias que faciliten el acceso y buen uso de la información (Amante, Costa y Extremeño, 2012); sin embargo, en este apartado se exponen servicios (Tabla 5) que no están en correspondencia con las ya conocidas habilidades de los usuarios actuales.

El hecho de que los servicios que se describen en la Tabla 5 no concuerden plenamente con las habilidades de los usuarios contemporáneos, acostumbrados al trabajo en línea o por medio de dispositivos electrónicos, se contradice con la sección dedicada a la formación de usuarios, donde se define el deber de la biblioteca de participar en la creación de una comunidad de aprendizaje, en la que los estudiantes encuentren de manera permanente las oportunidades y herramientas para desarrollar las competencias que les permitan hacer uso de la información, creando programas permanentes de información y asegurando el aprovechamiento de los recursos documentales para permitir conocer y actualizar datos básicos sobre las necesidades de información y hábitos de consulta.

\begin{tabular}{|l|l|}
\hline \multicolumn{1}{|c|}{ Servicios } & \multicolumn{1}{c|}{ Descripción } \\
\hline Horario continuo & $\begin{array}{l}\text { Servicios mínimo entre } 12 \text { y } 14 \text { horas diarias, con- } \\
\text { forme con el calendario escolar de la institución. }\end{array}$ \\
\hline Catálogo & Debe incluir todas las colecciones. \\
\hline
\end{tabular}




\begin{tabular}{|l|l|}
\hline Préstamo interno & $\begin{array}{l}\text { Otorgar dentro de los espacios de la biblioteca la } \\
\text { consulta de materiales. }\end{array}$ \\
\hline Préstamo externo & $\begin{array}{l}\text { Debe estar determinado por el reglamento 0 } \\
\text { políticas de la biblioteca. }\end{array}$ \\
\hline Préstamo interbibliotecario & $\begin{array}{l}\text { Es la posibilidad de hacer llegar los recursos } \\
\text { informativos fuera de la biblioteca. Esto debe estar } \\
\text { reglamentado para considerar el resguardo de los } \\
\text { materiales. }\end{array}$ \\
\hline Servicio de consulta o referencia & $\begin{array}{l}\text { Sustentado en colecciones formadas por recursos } \\
\text { informativos en diversos formatos y soportes, } \\
\text { actuales, pertinentes y fáciles de acceder y } \\
\text { recuperar. }\end{array}$ \\
\hline Consulta de recursos electrónicos & $\begin{array}{l}\text { Como una forma de hacer llegar a sus usuarios in- } \\
\text { formación actualizada, arbitrada y de fácil acceso. }\end{array}$ \\
\hline Formación de usuarios & $\begin{array}{l}\text { Ofrecer un programa permanente de formación } \\
\text { de usuarios dirigido a todos los miembros de la } \\
\text { comunidad. }\end{array}$ \\
\hline Reprografía & $\begin{array}{l}\text { Ofrecer la posibilidad de fotocopiar siguiendo las } \\
\text { normativas establecidas en materia de derechos } \\
\text { de autor y propiedad intelectual. }\end{array}$ \\
\hline
\end{tabular}

Tabla 5. Servicios para bibliotecas incluidos en las Normas para Bibliotecas de Instituciones de Educación Superior e Investigación

Pero estas no son las únicas normativas en México para las bibliotecas, pues existen leyes que protegen la actividad bibliotecológica y que forman parte del acervo a tener en cuenta para garantizar el funcionamiento de estas instituciones (Tabla 6).

\begin{tabular}{|l|l|}
\hline \multicolumn{1}{|c|}{ Leyes } & \multicolumn{1}{c|}{ Región } \\
\hline Ley General de Bibliotecas & Estados Unidos Mexicanos \\
\hline Ley de Fomento para la Lectura y el Libro & Baja California Sur \\
\hline Ley de Bibliotecas & Baja California Sur \\
\hline Ley de Bibliotecas & Guerrero \\
\hline Ley de Bibliotecas & Jalisco \\
\hline Ley de Bibliotecas & Quintana Roo \\
\hline Ley de Fomento a la Lectura & San Luis Potosí \\
\hline Ley del Libro y Bibliotecas Públicas & Durango \\
\hline Ley de Bibliotecas & Sinaloa \\
\hline Ley de Bibliotecas & Tlaxcala \\
\hline Ley de Bibliotecas & Chihuahua \\
\hline Ley del Libro y Bibliotecas Públicas & Tabasco \\
\hline
\end{tabular}




\begin{tabular}{|l|l|}
\hline Ley de Bibliotecas Públicas & Zacatecas \\
\hline Ley de Fomento para la Lectura y el Libro & San Luis Potosí \\
\hline
\end{tabular}

Tabla 6. Resumen de leyes para la actividad de bibliotecas en México

Las leyes enunciadas en la Tabla 6 pudieran ser una respuesta al hecho de que la estrategia del Estado mexicano hacia la actividad bibliotecológica no se encuentra asentada en la Constitución Política de los Estados Unidos Mexicanos (Cárdenas Zardoni, 2012). Esto genera una dispersión en la atención a las problemáticas que enfrenta el ámbito bibliotecológico a nivel nacional. ¿Cómo es posible desarrollar un reconocimiento social en todo el país de esta actividad si se realizan miradas individuales? No es objetivo de este trabajo profundizar en estas cuestiones, pero parece un tema que resultaría interesante a abordar en otro momento.

En el ámbito de la gestión documental, México ha realizado esfuerzos importantes. El 23 de enero de 2012 se expidió la Ley Federal de Archivos, publicándose la última revisión el 19 de enero de 2018. El 13 de mayo de 2014 se publicó el Reglamento a esta ley y el 15 de junio de 2018 se expidió la Ley General de Archivos (SEGOB, s. a.). De esa forma, se contribuye a la modernización y eficiencia de las actividades para brindar un acceso transparente a la información, de tal manera que permita su localización, disponibilidad e integridad. Es indispensable, además, que los documentos estén organizados y conservados con base en criterios uniformes.

La Ley Federal de Archivos, de obligatorio cumplimiento para los servidores públicos federales, define su objeto en su artículo 1 :

establecer las disposiciones que permitan la organización y conservación de los archivos en posesión de los Poderes de la Unión, los organismos constitucionales autónomos y los organismos con autonomía legal, así como establecer los mecanismos de coordinación y de concertación entre la Federación, las entidades federativas, los municipios y las demarcaciones territoriales de la Ciudad de México para la conservación del patrimonio documental de la Nación, así como para fomentar el resguardo, difusión y acceso de archivos privados de relevancia histórica, social, técnica, científica o cultural.

Las secciones de esta ley, descritas en la Tabla 7, abordan temas relacionados con la organización, administración, acceso y responsabilidades, los cuales pueden funcionar como estándares para minimizar la pérdida, destrucción u opacidad de información relevante.

\begin{tabular}{|c|l|l|r|}
\hline Secciones & \multicolumn{1}{|c|}{ Tema } & \multicolumn{1}{c|}{ Capítulos } & Artículos \\
\hline Título Primero & Disposiciones generales & Capítulo Único & Artículos 1 a 7 \\
\hline
\end{tabular}


IDENTIFICACIÓN DE NORMAS Y DIRECTRICES PARA BIBLIOTECAS...

\begin{tabular}{|c|c|c|c|}
\hline \multirow[t]{2}{*}{ Título Segundo } & \multirow{2}{*}{$\begin{array}{l}\text { De la organización y } \\
\text { administración de los } \\
\text { archivos }\end{array}$} & $\begin{array}{l}\text { Capítulo I. De la Organiza- } \\
\text { ción de los Archivos }\end{array}$ & Artículos 8 a 17 \\
\hline & & $\begin{array}{l}\text { Capítulo II. De la Adminis- } \\
\text { tración de los Archivos }\end{array}$ & Artículos 18 a 24 \\
\hline Título Tercero & $\begin{array}{l}\text { De los sujetos obligados } \\
\text { distintos del Poder Ejecu- } \\
\text { tivo Federal }\end{array}$ & Capítulo Único & Artículo 25 \\
\hline Título Cuarto & $\begin{array}{l}\text { Del acceso a los archivos } \\
\text { históricos y autorización } \\
\text { de salida y enajenación } \\
\text { de documentos }\end{array}$ & Capítulo Único & Artículos 26 a 34 \\
\hline \multirow[t]{2}{*}{ Título Quinto } & \multirow[t]{2}{*}{$\begin{array}{l}\text { Del Consejo Nacional } \\
\text { de Archivos }\end{array}$} & $\begin{array}{l}\text { Capítulo I. De la Integración } \\
\text { del Consejo Nacional de } \\
\text { Archivos }\end{array}$ & Artículos 35 a 38 \\
\hline & & $\begin{array}{l}\text { Capítulo II. Del Sistema } \\
\text { Nacional de Archivos }\end{array}$ & Artículos·39 a 40 \\
\hline \multirow[t]{5}{*}{ Título Sexto } & \multirow[t]{5}{*}{$\begin{array}{l}\text { Del Archivo General } \\
\text { de la Nación }\end{array}$} & $\begin{array}{l}\text { Capítulo I. De la operación } \\
\text { del Archivo General de la } \\
\text { Nación }\end{array}$ & \multirow[t]{3}{*}{ Artículos 41 a 48} \\
\hline & & $\begin{array}{l}\text { Sección Primera. } \\
\text { Del Órgano de Gobierno }\end{array}$ & \\
\hline & & $\begin{array}{l}\text { Sección Segunda. Del } \\
\text { Director General }\end{array}$ & \\
\hline & & $\begin{array}{l}\text { Capítulo II. Del Registro } \\
\text { Nacional de Archivos } \\
\text { Históricos }\end{array}$ & Artículos 49 a 51 \\
\hline & & $\begin{array}{l}\text { Capítulo III. De la Sección } \\
\text { de Archivos Presidenciales }\end{array}$ & Artículos 52 a 53 \\
\hline Título Séptimo & $\begin{array}{l}\text { De las infracciones } \\
\text { y sanciones }\end{array}$ & Capítulo Único & Artículos 54 a 56 \\
\hline
\end{tabular}

Tabla 7. Estructura de la Ley Federal de Archivos

Este tipo de documento constituye una poderosa herramienta para mejorar la gestión documental del patrimonio de cualquier nación, pues define un sistema para garantizar la durabilidad del patrimonio documental de un país.

\section{Metodología}

Los estudios que se realizan para abordar el tema de la normatividad en el ambiente bibliotecológico tienden a ubicarse en al menos tres áreas: por una parte, los trabajos que se enfocan en enunciar o describir el contenido de normas y leyes, sus principales aportaciones o vacíos (Albelda et al., 2014; 
Alsina, 2012; Balagué y Saarti, 2014; Cárdenas Zardoni, 2012; Giménez Chornet, 2015; González Guitián y Molina Piñeiro, 2008; López Lemos, 2016; Moreno Jiménez, 2005; Santovenia Díaz y Cañedo Andalia, 2006; Texidor, 2009); en otros casos, se declara el valor o impacto de la calidad en las actividades y procesos (Amante, Costa y Extremeño, 2012; Balagué y Saarti, 2014) o en la satisfacción de los usuarios (Arias, 2013; Arriola Navarrete, 2017; Balagué y Saarti, 2014), y en tercer lugar se aplican técnicas de recolección de datos para indagar sobre su viabilidad (Gaminde, Martínez Hervás y Yúfera Rodríguez, 2015).

Teniendo en cuenta estos aspectos, los objetivos de este trabajo se enfocaron en identificar los documentos normativos que regulan y orientan los procesos de las bibliotecas y las unidades de información, así como explorar la opinión de profesionales involucrados con la actividad bibliotecológica con respecto al uso de estándares en las unidades de información. Para cumplir el primer objetivo se llevó a cabo un análisis documental clásico, lo que posibilitó la familiarización teórica-conceptual así como establecer posibles conexiones entre las normas en el marco de la actividad bibliotecológica. Para el segundo objetivo se diseñó una encuesta como técnica de recolección de datos. De la aplicación de este instrumento se obtuvo la opinión de directivos y trabajadores de unidades de información con respecto al uso de normas y directrices.

La encuesta fue aplicada vía online por medio de grupos de discusión en LinkedIn, Facebook y correo electrónico. Las encuestas realizadas fueron 250. Del total de encuestas se utilizaron para el análisis 150 (60 \%) debido a que las restantes no fueron completadas.

Para el cuestionario se elaboró previamente una prueba piloto. Luego de obtener los resultados y realizar los ajustes formales necesarios, se diseñó un cuestionario con preguntas de opción múltiple lo cual permitió la selección de una opción de una lista de respuestas. Se utilizó además la escala de Likert, lo que permitió la selección de una única calificación dentro de posibles opciones.

Para indagar la opinión sobre el uso de las normas en el ambiente bibliotecológico, se tuvieron en cuenta cinco criterios: necesidad, aplicación, tipos, parámetros y evaluación (Tabla 8).

\begin{tabular}{|l|l|}
\hline Necesidad & Hecho que es necesario para alguien aplicar las normas \\
\hline Aplicación & Puesta en práctica de los procedimientos normativos \\
\hline Tipos & Modelo que sirve como pauta para clasificar los tipos de normas que se usan \\
\hline Parámetros & Elementos importantes a considerar en las normas \\
\hline Evaluación & Puesta en marcha de procesos de evaluación de normas aplicadas \\
\hline
\end{tabular}




\section{Presentación y análisis de resultados}

La distribución de los participantes (Figura 1) arrojó que, de los 150 encuestados, la mayor parte $(60 \%)$ ocupaba cargos directivos con una experiencia mínima de 5 años. En segundo lugar, con $25 \%$, estuvieron los especialistas en procesos técnicos. El tercer lugar lo ocupó el especialista en la prestación de servicio, con $15 \%$ de representatividad. Tanto especialistas de procesos técnicos como especialista de servicios poseían un máximo de 10 años de experiencia. El cuestionario incluyó la categoría especialista o responsable en procesos de calidad; sin embargo, no se obtuvieron respuestas.

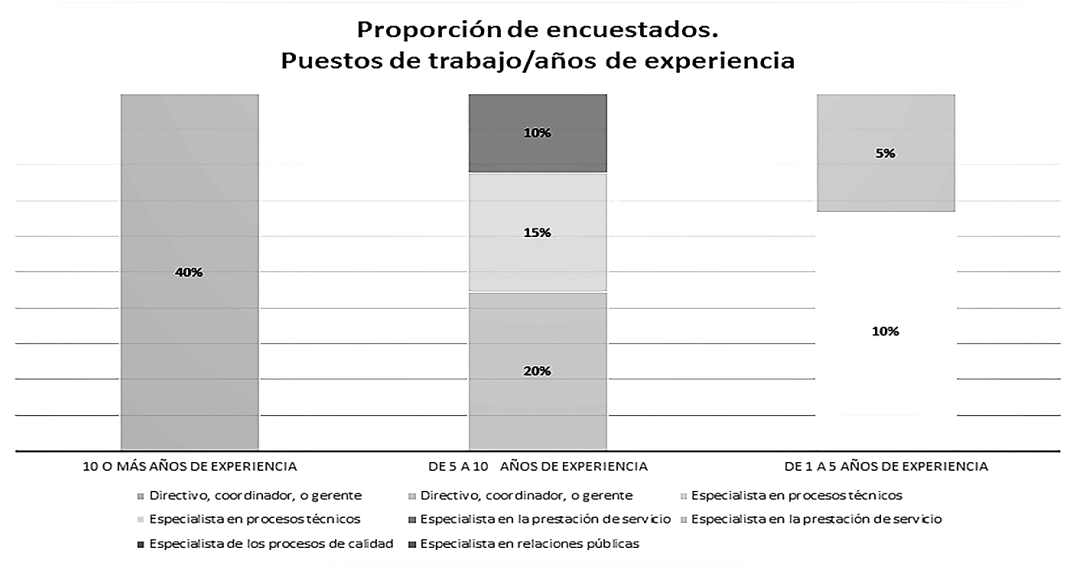

Figura 1. Proporción de encuestados

El tipo de unidad de información donde laboran los encuestados estuvo distribuido de la siguiente manera: bibliotecas especializadas, $30 \%$; centros de información, $32 \%$ y biblioteca universitaria, $26 \%$. En menor medida, los participantes pertenecían a bibliotecas escolares, $4 \%$; bibliotecas públicas, $4 \%$ y archivos, $4 \%$ (Figura 2 ).

En relación con la necesidad del uso de sistemas de normatividad o directrices en las unidades de información, $80 \%$ del total de encuestados contestó de manera positiva y $53 \%$ respondió que los aplican o los habían aplicado (Figura 3). Al analizar cada resultado, resalta que en archivos, bibliotecas públicas y bibliotecas escolares se observa menor distancia entre la necesidad de aplicar normas y su real aplicación (Figura 3).

Las bibliotecas universitarias, bibliotecas especializadas y centros de información obtuvieron los resultados más altos en expresar de manera positiva la necesidad de aplicar normas en las unidades de información. Sin embargo, 
al indagar si las han aplicado los niveles de respuesta disminuyeron en todos los casos.

\section{Proporción de tipos de organizaciones de información}

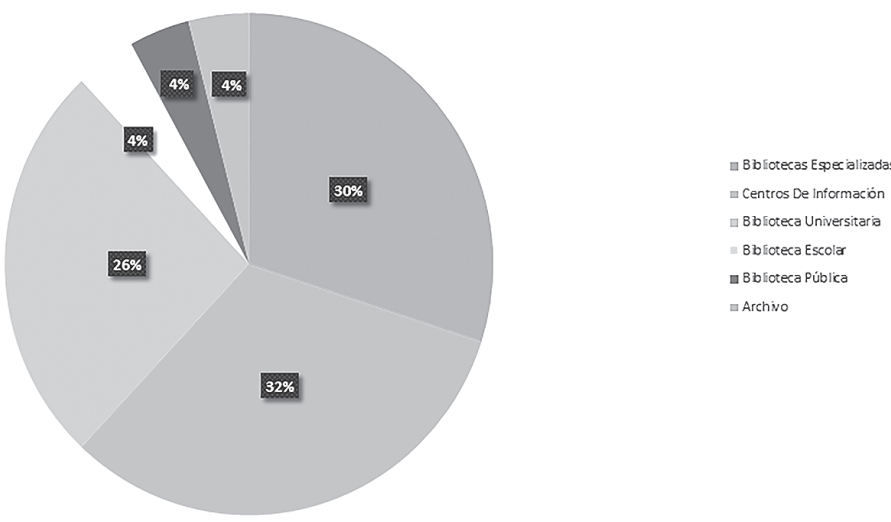

Figura 2. Proporción de tipos de centro de información donde laboran los encuestados

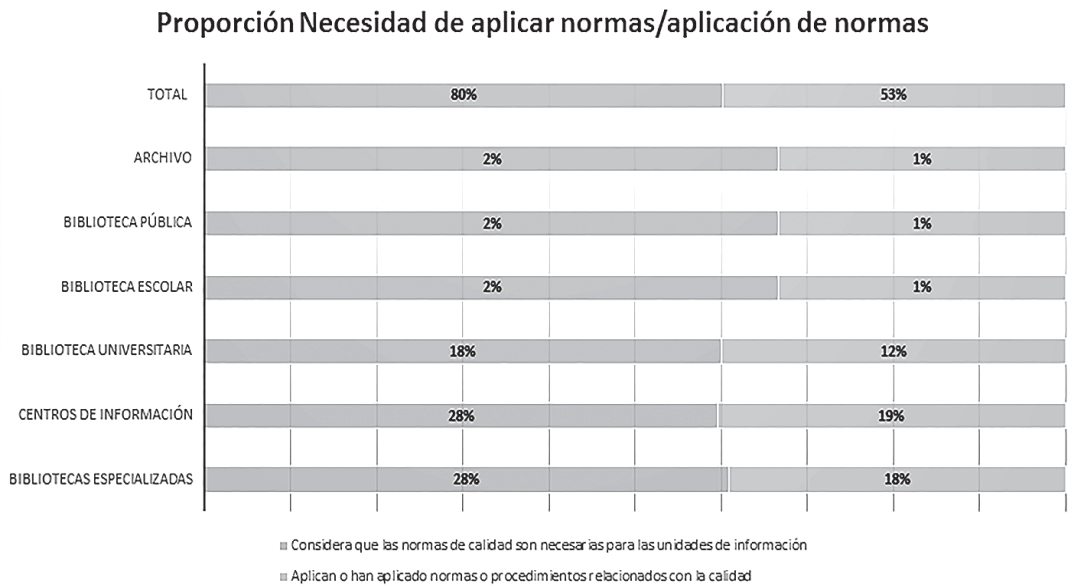

Figura 3. Diferencias entre necesidad de aplicar normas y aplicación de normas en unidades de información

$\mathrm{Al}$ abordar la relación entre estas dos variables mediante la aplicación del coeficiente de correlación, se obtuvo un resultado de 0.99928777919781 que indica una correlación positiva muy fuerte, pues cuando uno de los parámetros aumenta, el otro también lo hace en proporción constante. Este resultado es 
alentador para el uso de normas en unidades de información, pues se advierte una relación fuerte entre necesidad y aplicación de normas.

Del $53 \%$ de las unidades de información que señaló aplicar normas o directrices, $70 \%$, aplicó las normas ISO, 59 \% las normas de país y/o de la institución donde laboran y 30 \% las directrices de IFLA o de la ALA (Figura 4).

\section{Proporción de tipos de normas aplicadas}

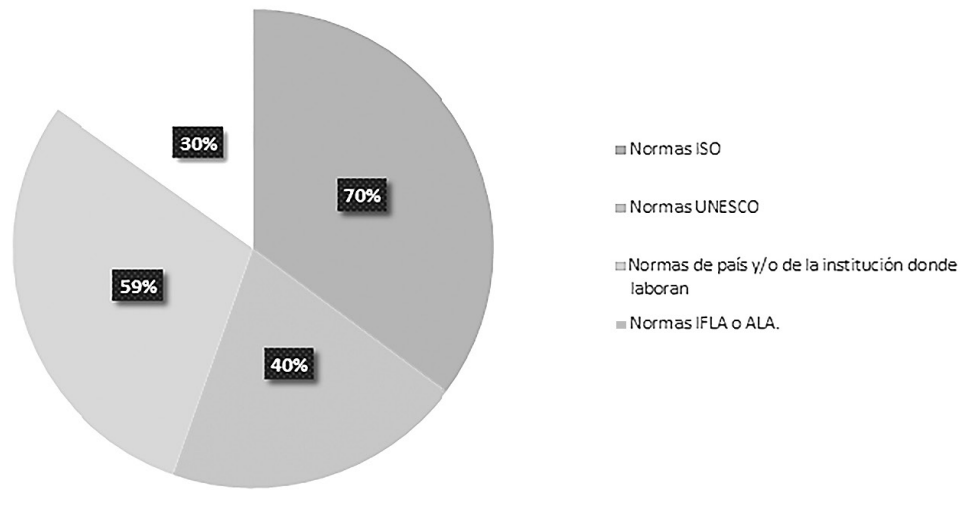

Figura 4. Proporción de tipos de normas aplicadas

El análisis del tipo de normas o directrices aplicadas por tipo de unidad arroja que $100 \%$ de las instituciones donde laboran los encuestados han aplicado un sistema, al menos una vez (Figura 5). Las bibliotecas universitarias, las bibliotecas especializadas, los centros de información y las bibliotecas públicas han aplicado al menos tres tipos de esquemas para estandarizar los procesos, mientras que los archivos y las bibliotecas escolares hasta dos. Las normas ISO y las directrices del país o de la institución donde laboran los encuestados fueron aplicadas por todos los tipos de unidades de información analizados.

En relación con los parámetros para la calidad que se propusieron a los encuestados, la mayor cantidad de respuestas estuvo entre totalmente de acuerdo y de acuerdo en que las normas deben considerar los siguientes parámetros: necesidades de información (96\%), habilidades de los usuarios $(87 \%)$, transparencia en la información (85\%), interconectividad $(74 \%)$, privacidad y protección de datos (66\%), acceso abierto a la información (64\%), tecnologías (58 \%) y educación en línea (57\%) (Figura 6). Con estos resultados, se reafirma la preocupación de los especialistas en las necesidades de los usuarios y cómo los nuevos temas que involucran a la actividad bibliotecológica están siendo tomados en cuenta para ser abordados de manera normativa. 


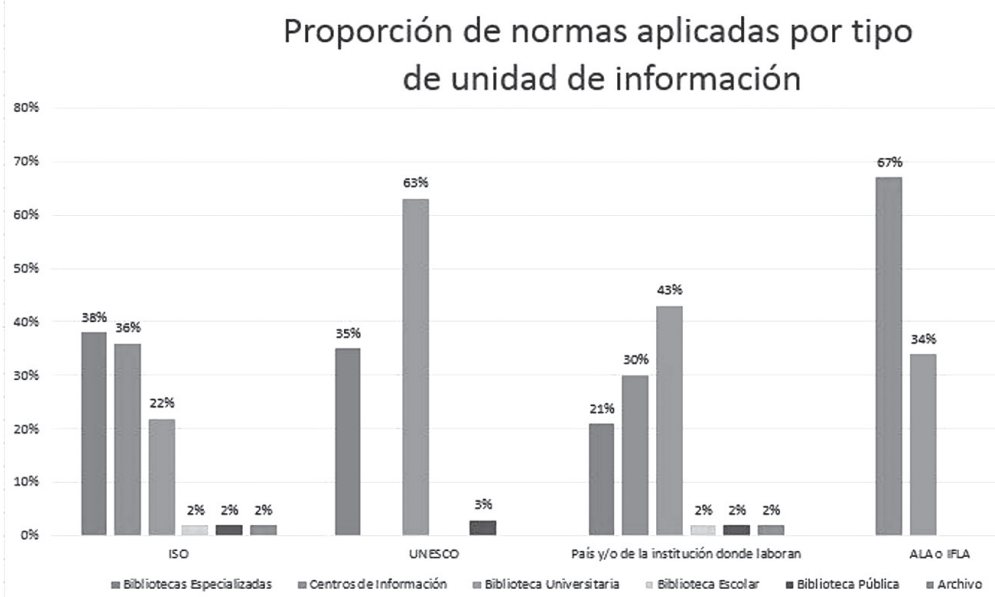

Figura 5. Proporción de tipos de normas aplicadas por tipo de unidad de información

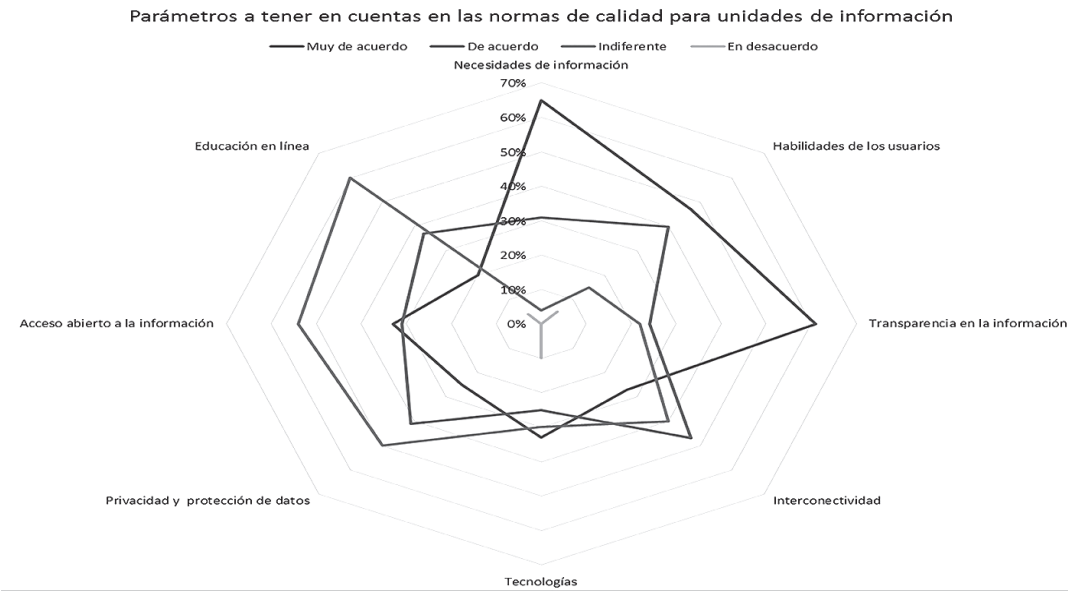

Figura 6. Proporción de los parámetros a tener en cuentas en las normas de calidad para unidades de información

Para este mismo criterio, un resultado interesante son los porcentajes que arroja la escala indiferente. En primer lugar se ubica el parámetro educación en línea (60\%), seguido por privacidad y protección de datos (50\%), y acceso abierto a la información (54\%). Este resultado pudiera ser un indicador de desapego de los encuestados para incluir estos temas emergentes en el contenido de las normas. 
En relación con la evaluación de las normas que se han aplicado en cada institución, $50 \%$ de las respuestas estuvo referida a que este proceso había ocurrido hacía menos de un año. Un porcentaje menor (40 \%) respondió que el sistema aplicado era coherente con los procesos de su organización y sólo $25 \%$ respondió que la aplicación de la norma había mejorado los procesos organizacionales. Estos resultados indican que los encuestados están familiarizados con el uso reciente de sistemas de normatividad y que para la mejora continua debe haber una coherencia entre la norma aplicada y los procesos organizacionales.

\section{Consideraciones finales}

Existen una gran cantidad de documentos que regulan los procesos de las unidades de información. Estos documentos son emitidos por organismos internacionales preocupados por la calidad en el desarrollo de estos procesos.

Las organizaciones ISO y Aenor son organismos normalizadores que no se dedican exclusivamente al ámbito de las unidades de información; sin embargo, han desarrollado una serie de documentos que orientan una amplia gama de procesos como organización y almacenamiento de documentos físicos y digitales, préstamos interbibliotecarios, evaluación y conservación de documentos, indicadores de rendimiento e infraestructura de edificios. De las normas desarrolladas por Aenor/ISO aplicables a las unidades de información, las siguientes resultan significativas por la gran amplitud de sus contenidos en diferentes rubros:

- Los procesos de desarrollo de indicadores:

- ISO 9001. Sistemas de Gestión de la Calidad

- ISO 2789. Información y documentación - Estadísticas internacionales de bibliotecas

- ISO 11620. Información y documentación - Indicadores de rendimiento de las bibliotecas

- UNE-50137. Información y documentación. Indicadores de rendimiento bibliotecario

- Para la gestión documental

- ISO 15489. Información y documentación - Gestión de documentos Parte 1 y 2

- ISO 30300. Información y documentación. Sistemas de gestión para los documentos. Fundamentos y vocabulario 
- Para el uso de metadatos en diferentes procesos

- UNE-ISO 23081. Información y documentación - Proceso de gestión de documentos. Metadatos para la gestión de documentos

- UNE-EN 15744. Identificación de películas. Conjunto mínimo de metadatos para trabajos cinematográficos

- UNE-ISO 15836. Información y documentación. Conjunto de elementos de metadatos Dublín Core

- Para los procesos de préstamos

- ISO 10161-1. Información y documentación - Interconexión de sistemas abiertos

- ISO 18626. Información y documentación - Las operaciones de préstamo entre bibliotecas

- Para la infraestructura

- ISO/TR 11219. Información y documentación - Condiciones cualitativas y estadísticas básicas para la construcción de bibliotecas

En sus manifiestos sobre la biblioteca pública y escolar, la Unesco define y potencia el papel de estas organizaciones en el fomento de la paz y la espiritualidad del ser humano. La IFLA y la ALA, por su parte, han centrado sus esfuerzos en temas que realzan el reconocimiento de las actividades que se realizan en el ambiente bibliotecológico, estableciendo políticas que representan la génesis de las bibliotecas.

En México, son varias las instituciones que se preocupan por establecer la normatividad de las unidades de información:

- El Consejo Nacional para Asuntos Bibliotecarios de las Instituciones de Educación Superior, A. C. de México (CONPAB-IES, A. C.) ha desarrollado normas para bibliotecas de instituciones de educación superior e investigación.

- Cada estado del país ha redactado y aplicado sus propias leyes para la actividad de bibliotecas, lo que por una parte solventa la necesidad de regularización pero por otra pudiera generar una dispersión en la atención a las problemáticas que enfrenta el ámbito bibliotecológico a nivel nacional.

- En la última década, México ha creado leyes encaminadas a potenciar la gestión documental del patrimonio de la nación. En 2012 se creó la Ley Federal de Archivo, en 2014 su reglamento, y en 2018 se emitió la Ley General de Archivos. Estos documentos constituyen una poderosa herramienta pues definen un sistema para garantizar la durabilidad del patrimonio documental. 
Es posible abordar el uso de normas en un cuestionario a partir de criterios como necesidad, aplicación, tipos, parámetros y evaluación. Entre los retos que se presentan para el uso de normas según los resultados obtenidos están los siguientes:

- Las normas se consideran como un proceso necesario a llevar cabo en unidades de información, siendo un aspecto que influye en la decisión de llevar a cabo su aplicación.

- El tipo de norma más utilizado es el ISO, en segundo lugar se encuentran las normas nacionales, en tercer lugar las directrices Unesco y por último las directrices de IFLA o de la ALA.

- Las instituciones que mencionaron aplicar normas utilizaron más de un sistema normativo y todas han incluido a las normas ISO como uno de estos sistemas.

- Las bibliotecas universitarias, las bibliotecas especializadas y los centros de información son unidades de información que aplican múltiples sistemas para normalizar-

- Las necesidades de los usuarios siguen siendo un parámetro determinante para la calidad de los procesos, por lo que deben ser una constante en el contenido de las normas.

- La transparencia en la información y la interconectividad constituyen dos parámetros emergentes que los encuestados mencionan que deben ser abordados en los documentos normativos.

- Parámetros emergentes como la educación en línea, la privacidad y protección de datos o el acceso abierto a la información no parecen ser, hasta el momento, temas necesarios a ser incluidos en el contenido de las normas.

- En relación con la evaluación de las normas aplicadas, los resultados arrojaron que el uso de la norma no garantiza una mejora de los procesos. Los resultados indican que para la mejora continua debe haber una coherencia entre la norma aplicada y los procesos organizacionales.

- Ningún encuestado especificó ocupar el cargo de especialista o responsable en procesos de calidad. Este resultado pudiera ser un indicio de la falta de esta figura en las unidades de información. 


\section{REFERENCIAS}

Aenor (Asociación Española de Normalización y Certificación). 2004. UNE-EN ISO 2789: Información y documentación. Estadísticas de bibliotecas para uso internacional. Madrid: Aenor.

Aenor. 2005. UNE-50137. Información y documentación. Indicadores de rendimiento bibliotecario. España: Aenor

Aenor. 2008. UNE 93200. Carta de servicios: requisitos. Madrid: Aenor.

Aenor. 2011. UNE-ISO 30300. Información y documentación: sistemas de gestión para los documentos: fundamentos y vocabulario. Madrid: Aenor.

Aenor. 2012. Directrices para la implementación de las normas 15744 y EN 15907. España: Aenor.

Albelda, E. B., G. M. Mano, M. M. M. Pérez y S. Romero. 2014. Nuevos instrumentos para la evaluación de bibliotecas: La normativa internacional ISO. Madrid: Aenor.

Alsina, M. G. 2012. "Contribución de la serie ISO 30300 a la gestión de la documentación judicial”. Ibersid 6: 135-143.

Amante, M. J., A. F. Costa y P. A. I. Extremeño. 2012. Una nueva biblioteca para una nueva universidad. Somonte-Cenero: Gijón.

Arias, C. A. 2013. Calidad de los servicios y satisfacción del usuario. Madrid: Facultad de Ciencias de la Documentación, Universidad Complutense de Madrid.

Arriola Navarrete, O. 2017. "Centro de Recursos para el Aprendizaje y la Investigación (CRAI): entorno dinámico de servicios”. Bibliotecas y archivos: órgano de la Escuela Nacional de Biblioteconomía y Archivonomía 2 (4): 16-29.

Ávila Barrientos, E. 2016. "Aplicaciones bibliotecológicas para el acceso abierto a la información científica en el entorno digital”. E-Ciencias de la Información 6 (2).

Balagué, N. y J. Saarti. 2014. Gestión de la calidad en la biblioteca: Diseña un sistema de gestión de la calidad basado en la norma ISO 9001. Barcelona: Editorial UOC.

Cárdenas Zardoni, H. 2012. "La biblioteca y la biblioteca escolar en la legislación educativa mexicana”. Biblioteca Universitaria 15 (2): 147-162.

CONPAB-IES y J. A. Verdugo Sánchez. 2012. Normas para bibliotecas de instituciones de educación superior e investigación. México: CONPAB/IES.

Gaminde, I., I. Martínez Hervás y B. Yúfera Rodríguez. 2015. "Indicadores de Calidad”, en XV Jornadas Nacionales de Información y Documentación en Ciencias de la Salud grupos de trabajo y pósteres: Bibliosalud 2014, editado por J. M. Estrada Lorenzo et al., 107-116. Madrid: Colegio Oficial de Médicos de Madrid.

Giménez Chornet, Vicent. 2015. "Normas ISO para la gestión de los documentos electrónicos: buenas prácticas para la gestión documental en las empresas". Fuentes, Revista de la Biblioteca y Archivo Histórico de la Asamblea Legislativa Plurinacional 9: 7-16.

González Guitián, María Virginia y Maricela Molina Piñeiro. 2008. "Las bibliotecas universitarias: breve aproximación a sus nuevos escenarios y retos". Revista Cubana de Información en Ciencias de la Salud 18 (2). Fecha de consulta: 23 de agosto de 2018. http://scielo.sld.cu/pdf/aci/v18n2/aci02808.pdf

IFLA (Federación Internacional de Asociaciones de Bibliotecarios y Bibliotecas). S. a. Manifiesto de las IFLA/Unesco sobre las Bibliotecas Digitales. Fecha de consulta: 20 octubre de 2016. http://www.ifla.org/ES/publications/manifiesto-de-las-ifla-unesco-sobre-las-bibliotecas-digitales 
IFLA. 1999. IFLA/Unesco School Library Manifesto 1999. Fecha de consulta: 20 octubre de 2016. https://www.ifla.org/node/7273

ISO. 2016. ISO 15489-1:2016 Information and documentation. Records management : Part 1: Concepts and principles. Geneva: ISO.

Ley Federal de Archivos. 2012. México: Cámara de Diputados. Fecha de consulta: 16 de julio de 2018. http://www.diputados.gob.mx/LeyesBiblio/pdf/LFA_150618.pdf

Linares Herrera, Manuel. 2010. "Se efectuó Congreso Mundial de Bibliotecas, IFLA 2010. Miradas al Acceso Abierto desde América Latina”. Ciencias de la Información 41 (3): 73-74.

López Lemos, Paloma. 2016. Novedades ISO 9001: 2015. Madrid: Fundación Confemetal.

Moreno Jiménez, Daniel. 2005. Interpretación de la norma ISO 9001: 2000 para obtener el certificado de calidad en bibliotecas. México: Colegio Nacional de Bibliotecarios.

Pacios, Ana R. 2016. "Universidades transparentes con bibliotecas transparentes". Investigación Bibliotecológica 30 (70): 105-128.

Pinto Molina, María y María de las Mercedes Fernández Valdés. 2010. “Alfabetización informacional, innovación y evaluación como funciones de la biblioteca universitaria del siglo XXI: visión desde un enfoque cualitativo". Ibersid 4: 81-91.

Rosas de Maidana, María Teresa. 2013. "Tecnología digital en Bibliotecas en Paraguay”. Revista e-Ciencias de la Información 3 (julio-diciembre): 1-18

Santovenia Díaz, Javier y Rubén Cañedo Andalia. 2006. "Norma UNE-ISO 15489-1: una guía para la gestión de los documentos de archivos". ACIMED 14 (5).

SEGOB (Secretaría de Gobernación). S. a. "Leyes y Reglamentos Federales”. Fecha de consulta: 23 de agosto de 2018. http://www.diputados.gob.mx/LeyesBiblio/

Texidor, Silvia. 2009. El desafío de certificar: la ISO 9001:2000 su aplicación en bibliotecas y otras organizaciones de servicio. Rosario: Nuevo Paradigma.

Unesco (Organización de las Naciones Unidas para la Educación, la Ciencia y la Cultura). S. a. Manifiesto de la Unesco sobre la biblioteca pública. Fecha de consulta: 1 septiembre de 2016. https://unesdoc.unesco.org/ark:/48223/pf0000112122_spa

Vargas Echeverría, Shilia Lsset. 2017. "Bibliotecas verdes: ¿Existen en Yucatán?”. Biblioteca Universitaria 20 (enero-junio): 35-46.

Para citar este texto:

Nieves Lahaba, Yadira Rosario. 2019. "Identificación de normas para bibliotecas: las unidades de información”. Investigación Bibliotecológica: archivonomía, bibliotecología e información 33 (78): 81-103. http://dx.doi.org/10.22201/iibi.24488321xe.2019.78.57864 